\title{
AC 2012-4347: A CUSTOM-PCB DESIGN FOR MICROCONTROLLER EDUCATION
}

\section{Mr. Ryan Andrew Taylor, University of Alabama}

Ryan Taylor received his B.S. in electrical and computer engineering in 2008 and his M.S. in 2011, both at the University of Alabama in Tuscaloosa, Ala. He is currently working toward his Ph.D. in computer engineering at Mississippi State University, Starkville, Miss. His research interests include microcontroller-based systems, sensor networks, image processing, and VLSI design.

\section{Dr. David Jeff Jackson, University of Alabama}

David Jackson received his B.S. in physics (1984) and M.S. in electrical engineering (1986) from Auburn University. He received his Ph.D. in electrical engineering (1990) at the University of Alabama. He is currently a professor of electrical and computer engineering at the University of Alabama. Jackson is a Senior Member of the IEEE and ISCA and a member of the IEEE Computer Society and ASEE. He has served on the Board of Directors for the International Society for Computers and their Applications and has served as Editor-in-Chief for the International Journal of Computers and their Applications. Additionally, he has served on the program committee and organizing committees for multiple international conferences. Jackson has more than 70 journal and conference publications, has directed the research of more than 30 graduate students, and served as Principal or Co-principal Investigator for research projects totaling approximately $\$ 7$ million in research expenditures. Jackson's current research interests include image processing, embedded systems, sensor and sensor network development, VHDL hardware description language, and programmable device technologies. 


\section{A Custom-PCB Design for Microcontroller Education Background and Motivation}

Much time and effort has been spent attempting to discover the best approaches to the problem of microcontroller education. The methods and processes of teaching microcontrollers are important components to the education of potential electrical and computer engineers. Across the variety of institutions that teach these concepts, the methods differ. However, the main focus and outline of the topics that are necessary to cover at this level stay the same.

During a traditional prerequisite flow for computer engineering students in an undergraduate curriculum, students tend to study microcontroller topics in a standard order and transition. At The University of Alabama, students begin their work toward designing microcontroller systems in ECE 285, which covers C programming. Students then continue their work toward microcontroller systems in the ECE 380 course. This course covers such topics as number systems, Boolean algebra, and combinational and sequential logic systems ${ }^{1}$. This course lays the groundwork and prepares the student for the first microcontrollers course that is labeled ECE 383. This course covers microcontrollers, assembly-language programming, interrupts, hardware interfacing, and other related topics. Beyond these courses, the students are expected to be familiar enough with microcontrollers and peripheral interfacing topics to put their skills into practice in courses such as ECE 480, ECE 484, and ECE 486, which discuss microcontroller systems at a much more complex level ${ }^{2,3}$.

This is the traditional format and progression of a normal undergraduate curriculum of electrical and computer engineering education. This progression is fairly rigid, due to the fact that students must master this introductory material in order to be successful with more complex microcontroller systems. In the Department of Electrical and Computer Engineering at Iowa State University, the first course that is taken by undergraduate students is at the sophomore-level called CPRE 211: Microcontrollers and Digital Systems Design. This course covers embedded hardware models, embedded programming at the $\mathrm{C}$ and assembly levels, and $\mathrm{I} / \mathrm{O}$ interfaces of peripheral devices. Only then does the student move on to the more difficult courses of CPRE 488 and CPRE 588, Embedded Systems Design and Embedded Computer Systems, respectively, with the latter being a graduate level course. These courses cover material that requires the prerequisite material of the sophomore-level course, very similar to the relationship between ECE 383 and the senior-level courses ECE 480 and ECE 484 at our institution ${ }^{1,2,3,4}$.

As discussed in this document, as well as in another article ${ }^{5}$, the upper-level courses assume a sufficient background in the subjects relating to hardware and peripheral interfacing, so these topics must be covered at the beginning of the computer engineering sequence of an electrical and computer engineering curriculum. These introductory courses familiarize the students with semi-complex microcontroller systems and their design and construction. The students then enter the terminal courses with a good foundation from which to work.

The issue of difficulty in this sequence of topics being used comes when the student is faced with the task of working through laboratory assignments that cover assembly language programming, to some complexity, and microcontroller-peripheral interfacing at the same time. In Fall of 2003, Mississippi State University began a process to address this issue. Previous to this time, the 
introductory microcontrollers course was set up in this fashion, with a heavy emphasis on assembly language programming. The course was modified to discuss assembly coding, but to have the majority of the focus of the topics geared toward embedded system concepts and design prototyping, including hardware interfacing, while using $\mathrm{C}$ programming as the major language of choice. By incorporating a focus such as this, more complex interfacing assignments are able to be completed by the students as laboratory assignments. In the words of the researchers ${ }^{7}$, "grasping the hardware interfacing issues is difficult enough without adding the extra complexity of coding applications in assembly language."

To begin building and designing a course, the proper hardware must be selected to tailor the students' assignments. In many cases ${ }^{7}$, a large microcontroller unit (MCU) development kit is selected that includes the microcontroller and all peripheral devices on board. This is the design format that the Department of Electrical and Computer Engineering at The University of Alabama has been using in previous years. The Dragon12-Plus MCU from EVBplus has been the main hardware that laboratory assignments for ECE 383 have been built around ${ }^{2,3,8}$. This has contributed to a decline in the grasp of the fundamentals of microcontrollers and peripheral interfacing. Possible reasons could include the absence of the connections in physical sight of the user when working with the MCU, or possibly the sheer magnitude of the device that the student is working with. The fact that the students working the assignments have not been making the physical wiring connections from the microcontroller to the peripheral device(s), because of the monolithic nature of the MCUs, may be contributing to this decline in peripheral interfacing aptitude. If the students were required to make these connections themselves, it is possible that a more fundamental understanding of these topics would be obtained.

This document introduces the solution that we are attempting to implement into our undergraduate electrical and computer engineering curriculum. The solution is a daughter card that will connect directly to the NI ELVIS workstation and, thus, allow access to the entirety of the instrumentation suite offered by it. This card, called the Microcontroller Unit Peripheral Card (MCUPC), attempts to address the issues that are facing potential engineers in need of a solid instruction in microcontrollers and peripheral interfacing, without coming at the expense of familiarity with advanced assembly programming techniques.

The MCUPC is a printed circuit board (PCB) that inserts into the prototype area of the NI ELVIS workstation and can fully connect to the functions and tools that are offered by the workstation suite. The MCUPC is built as a workbench for a traditional laboratory setting that is used with a course that is similar to the one in question. It contains sockets for a microcontroller in a 28-pin (or smaller) dual-inline package (DIP) as well as several components of various sizes. A standard set of integrated circuits (ICs) that comprise a basic introduction to peripheral interfacing is included as a suggested group of components that comprise the MCUPC laboratory system. 


\section{Implementation}

The Dragon12-Plus MCU from EVBplus/Wytec is the trainer module that has been used previously for the laboratory instruction in the ECE 383 Microcomputers course. This laboratory instructional system has been used to teach assembly language programming, peripheral interfacing, and modular system design. The system itself is a 16-bit MCU designed and built for an academic environment.

Several factors went into the choice of what microprocessor to include in the MCUPC laboratory system design. First, and, arguably, most importantly, the resultant addition to the cost of the entire system must be taken into account. With an instructional laboratory system such as this, reducing the cost of each component as much as possible is important. In the case that the institution provided this component, or in the case that the user was asked to provide this component individually, the cost must be a manageable amount.

Functionality is also an important factor. A microprocessor or microcontroller unit must be selected that implements the functions necessary to meet the needs of the specific laboratory course unit for which the system is being used. The MCUPC is intended to meet the needs of a course covering the basics of microcontrollers and peripheral interfacing, thus the MCU must provide the capability of handling and working with multiple communication methods and types of interfaces.

A third important factor for choosing the microprocessor unit is the flexibility of the physical footprint trading off with the number of bidirectional I/O pins that are available in a hardware package implementation. Because of the size limitations of the MCUPC, the physical footprint of any component that is selected is considered so that the largest set of components possible can be included with the system. Also, the number of $\mathrm{I} / \mathrm{O}$ pins available in a specific package is important because of the extensive peripheral interfacing done with this system.

Based on all factors considered for the choice of the MCU to be used in the MCUPC laboratory instructional system, the PIC24HJ64GP502 microprocessor from Microchip Technologies was selected. This microprocessor development kit is USB-powered and includes an on-board debugger and programmer using this connection. The Microstick can be used with the aforementioned processor or any other processor that can be manufactured in a 28-pin SPDIP. The development kit, or the microprocessor alone, can be used with the MCUPC as there are adequate sockets to support them both.

When designing the MCUPC, a major factor in component and peripheral selection was to obtain a set of components that would allow a wide range and variety of interfaces to be used in the instructional process. For every component that was used in the ECE 383 course to instruct some interfacing concept, a corresponding component was selected. Because of this design specification, the same instruction can take place in an environment more conducive to microcontroller instruction.

The headers selected are manufactured by Sullins Connector Solutions. All headers used on the MCUPC rise above the PCB at the same height. Uniformity keeps the wiring that will be 
completed by the user during laboratory assignments as clean as possible ${ }^{15}$. By using the virtual instrumentation suite of the NI ELVIS system via these connection points, the MCUPC offers a wider capability of functionality than it would otherwise. The user is given access to a toolsuite that can be used throughout the design and prototyping process.

To provide some usefulness to the user of the laboratory system, a prototyping area must be afforded to the user. In the case that the institution wishes to implement an assignment or project involving a set of components that are not included with the original MCUPC, a breadboarding area is provided for the user to make use of at his or her discretion. The solderless breadboard that was chosen to be implemented with the MCUPC is the 270-Point Breadboard item manufactured by Pololu ${ }^{16}$. There are 23 rows of pins allowing the use of several different configurations of ICs. If an institution desired to use a microprocessor with this system that was not available in a 28-pin (or smaller) SPDIP, it could be conveniently mounted on the prototyping area and used appropriately.

As one of the methods of receiving input from the user when utilizing the MCUPC, a $3 \times 4$ matrix output keypad is provided. Futurlec Electronic Components offers a Small Keypad that boasts a small physical footprint of 2.008 inches by 2.520 inches. Using this matrix output from the keypad, the user of the laboratory system will be required to create semi-complex algorithms to handle button presses, debouncing, and input priority, among other tasks.

The MCU of choice for the MCUPC is the Microstick development kit manufactured by Microchip. The Microstick, which makes use of the PIC24HJ64GP502 microprocessor $^{14}$, has sixteen remappable pins for use with input and output. However, only seven of these pins are five volt tolerant. Because a larger quantity of input and output pins will frequently be necessary, a separate IC is implemented to offer this functionality. Also, the pin expander chosen uses an $\mathrm{I}^{2} \mathrm{C}$ interface, which allows the user an avenue for using this method of communication and interfacing ${ }^{14}$. The IC is called the MCP23017 16-Bit I/O Expander with Serial Interface from Microchip Technologies. The Microstick MCU development kit has built-in support for the $\mathrm{I}^{2} \mathrm{C}$ interface so that the user is not responsible for the physical implementation of the clock and data signal. It is instructional for a student to work with this interface because a fundamental knowledge of the communication is necessary for successful operation; however, the special function registers of the $\mathrm{I}^{2} \mathrm{C}$ module allow easy and direct access to all control and status operations of this method of communication.

One of the methods of sending data to a peripheral device is through pulse-width modulation (PWM). More specifically, PWM is frequently used to control power distribution to a motor. The use of PWM in an instructional environment such as a microcontrollers course is necessary due to the requirements of controlling such a signal. The calculations made, and also the hardware requirements, help the user in his or her understanding of peripheral device control.

The PWM-controlled fan that was selected is the FFB0412VHN-TP03 fan by Delta Electronics. The fan itself has a rated voltage of twelve volts DC and an average speed of approximately 9500 revolutions per minute. This fan was chosen because of its relatively small physical footprint over a similar counterpart that has a physical footprint exactly twice its size. 
The fan has four outputs provided over independently insulated leads. These inputs are colorcoded for ease of use. The black wire is associated with the negative voltage and the red wire is associated with the positive voltage. The blue wire provides the user with a tachometer output, which gives a number of pulses based on the number of rotations of the fan, and the yellow wire is the PWM control, effectively controlling the speed of rotation according to its duty cycle. Further information regarding these signals can be found in the manufacturer's datasheet ${ }^{19}$.

A motor driver is a component that is often used in a laboratory environment regarding microcontroller education. A half-H driver is an IC that accepts inputs at TTL- and low-level CMOS logic levels and uses them to produce and control high-current and/or high-voltage loads. When dealing with motor applications in a peripheral device-based course, these drivers are a necessity. The IC that was selected for this application is the SN754410 Quadruple Half-H Driver from Texas Instruments. This particular component is used in a sixteen-pin DIP.

Because there are so many different types of components loaded on the MCUPC, and due to the lack of physical footprint space on the $\mathrm{PB}$, only a subset of the voltages required can be made available in regulated voltage form. A $5 \mathrm{~V}$ regulator was selected to provide the user with the capability of making use of a simple voltage regulator component package and providing a signal that could be used with many basic components. Since the NI ELVIS workstation, through the mounting headers on the outer rim of the MCUPC, offer $+15 \mathrm{~V}$ and $+5 \mathrm{~V}$ as always-available connections, the $+5 \mathrm{~V}$ regulator is merely a tool to assist the user when working with voltage regulators in a learning environment. $\mathrm{A}+3.3 \mathrm{~V}$ regulator is also provided so that the microprocessor could be used separate and apart from the Microstick development kit, if necessary or desired ${ }^{20}$.

A Digital-to-Analog convertor (DAC) is a component that can be used for a myriad of different applications, especially those in the setting of a course covering microcontrollers and peripheral interfacing. For audio signals and other applications, the DAC IC selected can be used for instructional purposes. Maxim Integrated Products offers the MAX548ACPA+ eight-bit lowpower SPI DAC in an eight-pin DIP. This component package was selected because of its availability and its relatively small physical footprint. The component has one voltage output associated with the lone data converter and uses a single-supply voltage source ${ }^{22}$.

Electrically Erasable Programmable Read-Only Memory (EEPROM) is a particular implementation of non-volatile memory that is used to retain small amounts of data in microcontroller systems. The interfacing of this particular component gives the user another look at the $\mathrm{I}^{2} \mathrm{C}$ interface for peripheral control. Microchip Technologies manufactures the 24LC515$\mathrm{I} / \mathrm{P}$, a $400 \mathrm{kHz}$ frequency EEPROM device that has an input voltage range of $2.5 \mathrm{~V}$ to $5.5 \mathrm{~V}$. This particular component is available in an eight-pin DIP IC that is mounted very similarly to the other components on board the MCUPC ${ }^{23}$.

One of the most widely used forms of digital output, with regard to microcontroller systems, is the Liquid Crystal Display (LCD) module. The character LCD screen normally has a proprietary input method that would not be familiar to the user. This makes it a helpful addition to any laboratory or educational system that is designed. It is also an interesting peripheral that is very popular in microcontroller courses. 
The LCD module that was chosen is the Crystalfontz America CFAH1602B-TMI-JT Character LCD Module. The classification information from the product number gives information from the manufacturer's datasheet ${ }^{24}$. The module is a sixteen character by two line character LCD display. It uses a white light-emitting diode (LED) backlight and works with multiple font sets.

To implement the MCUPC, a suitable PCB design software suite was chosen. PCB Artist is a software program from Advanced Circuits that functions as a PCB layout system and schematic capture suite. The software suite includes tools for schematic capture as well as a PCB layout system that is integrated tightly with the circuit schematics in an associated project. The ordering process is also a feature that is incorporated into the design suite ${ }^{25}$.

Several design specifications must be considered when designing a system such as the MCUPC. First, the connection headers provide the same access to the instrumentation suite that the NI ELVIS itself would. Thus, no advantage of the workstation is lost. The MCUPC is built to the exact physical dimensions as the NI ELVIS' workbench so that it can also mount the board in place. When designing this hardware, one of the main objectives was to design and assemble a system that would be flexible enough to meet the needs of most laboratory and instructional settings in which it would be used. To achieve this goal, the sockets and housing of the microprocessor unit can be employed in two different ways. In Figure 1, the completed MCUPC is shown. Toward the bottom left is the housing for the MCU, itself. The microprocessor could be inserted into the innermost headers, or the Microstick PCB kit can be inserted into the outermost headers. Also, since the MCUPC is resting on top of the NI ELVIS workstation's breadboarding area, a sufficient area needed to be afforded to the user on the MCUPC PCB.

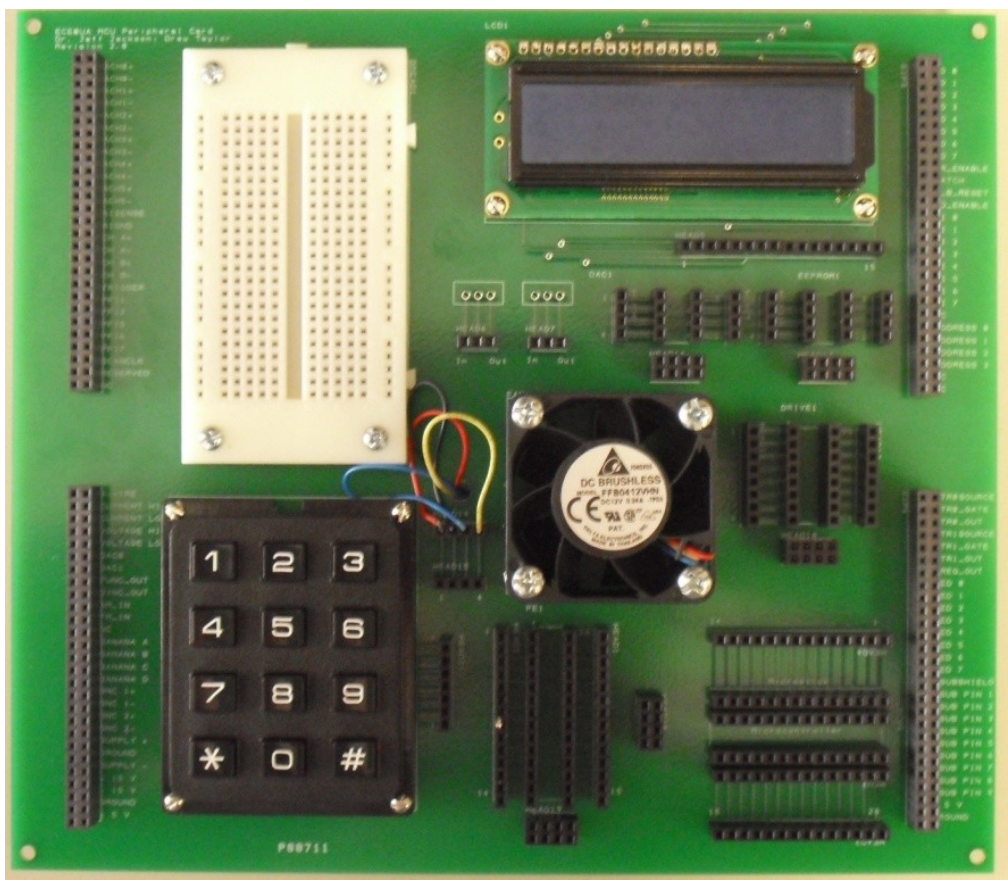

Figure 1. Fully populated MCUPC PCB. 


\section{Implementation into a curriculum and measurement of results}

The implementation of this system includes the purchase of all materials, the construction and assembly of the PCB by the course administrator(s), and the compilation of the laboratory assignments associated with the system. This process can be completed in a reasonable amount of time and with a reasonable amount of funds. All minor components of this MCUPC laboratory system can be obtained readily from most electronic distributors. The others are easily obtained from their respective manufacturers.

The cost breakdown of all components needed for the MCUPC system to be implemented (not including the NI ELVIS workstation) is shown in Table 1. To reduce the unit cost of the components, and in order to estimate the cost of implementing the MCUPC system into a full laboratory setting, it is assumed that a quantity of components sufficient to construct five units is needed. It can also be noted that nearly all materials that are required for a laboratory assignment, and not physically located on the MCUPC itself, are not included in this compilation. The total cost of all items is estimated to be $\$ 1063.14$.

Table 1. Cost breakdown of components for five MCUPC laboratory systems.

\begin{tabular}{|c|c|c|c|}
\hline Component & Vendor/Distributor & Quantity & Total Cost \\
\hline Custom PCB & Advanced Circuits & 5 & $\$ 333.63$ \\
\hline Breadboard & Pololu & 5 & $\$ 16.50$ \\
\hline Keypad & Futurlec & 5 & $\$ 11.00$ \\
\hline LCD Module & Crystalfontz & 5 & $\$ 114.80$ \\
\hline Regulator $(+3.3 \mathrm{~V})$ & Digikey & 5 & $\$ 9.90$ \\
\hline Regulator $(+5 \mathrm{~V})$ & Digikey & 5 & $\$ 8.85$ \\
\hline DAC & Digikey & 5 & $\$ 30.45$ \\
\hline EEPROM & Digikey & 5 & $\$ 19.70$ \\
\hline Fan & Digikey & 5 & $\$ 72.50$ \\
\hline Driver & Digikey & 5 & $\$ 10.70$ \\
\hline Microstick kit & Microchip & 5 & $\$ 124.95$ \\
\hline 23017 Expander & Digikey & 5 & $\$ 7.20$ \\
\hline $2 \times 27$ header & Digikey & 20 & $\$ 160.20$ \\
\hline $1 \times 3$ header & Digikey & 10 & $\$ 4.20$ \\
\hline $1 \times 4$ header & Digikey & 50 & $\$ 26.00$ \\
\hline $1 \times 8$ header & Digikey & 20 & $\$ 15.00$ \\
\hline $1 \times 7$ header & Digikey & 5 & $\$ 3.40$ \\
\hline $1 \times 14$ header & Digikey & 50 & $\$ 58.00$ \\
\hline $1 \times 16$ header & Digikey & 5 & $\$ 6.25$ \\
\hline $2 \times 4$ header & Digikey & 25 & $\$ 21.25$ \\
\hline $2-56$ screw package & Radio Shack & 1 & $\$ 4.28$ \\
\hline $4-40$ screw package & Radio Shack & 1 & $\$ 4.38$ \\
\hline
\end{tabular}

For the four introductory example laboratory assignments that might be used with a system such as this, the hardware interaction is very minimal. These assignments require the user to complete simple tasks requiring either the NI ELVIS by itself or no hardware devices at all. The advanced 
laboratory assignments that follow move the user into a further level of complexity when dealing with the set of peripherals that are loaded with the MCUPC.

The fifth laboratory assignment addresses and reinforces the user's knowledge of ANSI-standard $\mathrm{C}$ programming and introduces the student to working with the Microstick in conjunction with the MCUPC and the NI ELVIS Virtual Instrumentation Suite. The user is required to use the Microstick development kit to create a simple digital system that flashes an LED on board the device. The sixth laboratory assignment introduces the user to the design and prototyping of digital systems that implement slightly more complex input and output than Lab 05 . The user works with polled I/O and interrupt-driven I/O in this assignment to meet the goal of designing more complex systems. This assignment requires the user to first implement a simple polled I/O system. This system utilizes the MCUPC, the Microstick, and the NI ELVIS system. The polls a pushbutton and delivers output via an LED on the NI ELVIS system. Then the user is required to implement a system with similar functionality using an interrupt-based design. The seventh assignment introduces the user to the use of serial communication using the Serial-Peripheral Interface (SPI) using the MAX548A DAC and the Inter-Integrated Circuit $\left(\mathrm{I}^{2} \mathrm{C}\right)$ interface using the 24LC515 EEPROM. The eighth laboratory assignment allows the user to obtain more experience and a working knowledge of more advanced I/O-intensive applications. The twelvebutton keypad and character LCD module are used in this assignment to further the student's ability to work with semi-complex I/O systems. The ninth, and final, assignment introduces the user to the topic of driving motors and fans using the Microstick and the MCUPC. The user makes use of a motor driver supplied as a peripheral to drive a high-current DC motor and a four-wire PWM signal to drive a DC fan, included with the MCUPC system. The advanced laboratory assignments allow the student to gradually introduce themselves to the components that are included with the system. All components are covered with this set of laboratory assignments that are built around the MCUPC.

The overall goals of this research are to enhance a user's laboratory experience in an undergraduate course that discusses microcontrollers and their associated peripheral interfacing capabilities. There are multiple ways to assess this measure. The most obvious and telling of these are from specific assignments and associated assessments in not only the aforementioned course, but also subsequent courses that make use of microcontrollers and peripheral devices. At this institution, the subsequent courses in question are labeled as ECE 480/481, ECE 484, and ECE 486/487. All of the topics covered in these courses require the prerequisite knowledge of microcontrollers and peripheral interfacing ${ }^{2,3}$.

In order to assess these measures accurately, a set of data from these courses would need to be acquired. Ideally, assessments from these courses could be retrieved for a two- or three-semester period to serve as a control section of data. After this is obtained, the set of students' grades could be obtained from the courses after the MCUPC laboratory system has been implemented. Using this data, it would be evident whether the inclusion of the MCUPC into the laboratory curriculum was beneficial for the users and the institution's educational goals. 
[1] University of Alabama Undergraduate Catalog, The University of Alabama, Tuscaloosa, AL, 2010.

[2] The University of Alabama. (2011, Sep.) Electrical Engineering Program 2010 Curriculum Flowchart. [Online]. Available: http://ece.eng.ua.edu/files/2011/07/EE_Flowchart_2010.pdf

[3] The University of Alabama. (2011, Sep.) Computer Engineering Option 2010 Curriculum Flowchart. [Online]. Available: http://ece.eng.ua.edu/files/2011/07/CEO Flowchart 2010.pdf

[4] Bezdek, M.; Helvick, D.; Mercado, R.; Rover, D.; Tyagi, A.; Zhao Zhang; , "Developing and Teaching an Integrated Series of Courses in Embedded Computer Systems," Frontiers in Education Conference, $36^{\text {th }}$ Annual, pp. 19-24, 27-31 Oct. 2006 doi:

10.1109/FIE.2006.322749 URL: http://ieeexplore.ieee.org/stamp/stamp.jsp? $\mathrm{t} \mathrm{p}=$ \&arnumber $=4117237 \&$ isnumber $=4116830$

[5] Chin-Ming Hsu; Hui-Mei Chao; , "An Autonomous Mobile Robot System for Advanced Microcontroller Education," INC, IMS, and IDC, 2009. NCM '09. Fifth International Joint Conference on , pp. 1709-1714, 25-27 Aug. 2009 doi: 10.1109/NCM.2009.287

URL: http://ieeeexplore.ieee.org/stamp/stamp.jsp? t $\mathrm{p}=$ \&arnumber $=5331305 \&$ isnumber $=5331299$

[6] Jianyun Ni; Jing Luo; , "Microcontroller-based engineering education innovation," Educational and Information Technology (ICEIT), 2010 International Conference on , vol.3, pp. V3-109-V3-112, 17-19 Sept. 2010 doi: 10.1109/ICEIT.2010.5608413 URL: http://ieeeexplore.ieee.org/stamp/stamp.jsp? tp $=$ \&arnumber $=5608413$ \&isnumber $=5607525$

[7] Reese, R.B.; Jones, B.A.; , "Improving the effectiveness of microcontroller education," IEEE SoutheastCon 2010 (SoutheastCon), Proceedings of the , pp. 172-175, 18-21 March 2010 doi: 10.1109/SECON.2010.5453984 URL: http://ieeexplore.ieee.org/stamp/stamp.jsp? $\mathrm{t} \mathrm{p}=$ \&arnumber $=5453894 \&$ isnumber $=5453808$

[8] EVBplus / Wytec. (2011, Sep.). The Dragon12-Plus-USB. [Online]. Available: http://www.evbplus.com/9s12/9s12 hes $12 . h t m l$

[9] Grupta, G.S.; Chew Moi-Tin; , "New Frontiers of Microcontroller Education: Introducing SiLabs ToolStick University Daughter Card," Sensor Networks, Ubiquitous and Trustworthy Computing, 2008. SUTC '08, IEEE International Conference on , pp. 439444, 11-13 June 2008 doi: 10.1109/SUTC.2008.35 URL: http://ieeeexplore.ieee.org/stamp/stamp.jsp?tp= $\$$ arnumber $=4545799$ \&isnumber $=4545723$

[10] Deaky, B.; Lupulescu, N.B.; Ursutiu, D.; , "Extended educational use of the Microcontroller Student Learning Kit (MCU SLK)," Global Engineering Education Conference (EDUCON), 2011 IEEE , pp. 913-916, 4-6 April 2011 doi: 10.1109/EDUCON.2011.5773254 URL: http://ieeexplore.ieee.org/stamp/stamp.jsp? $\mathrm{tp}=$ \&arnumber $=5773254 \&$ isnumber $=5773100$

[11] Reese, Robert, J.W. Bruce, and Bryan A. Jones, Microcontrollers: From Assembly Language to C Using the PIC24 Family. Boston, MA: Course Technology, 2009.

[12] National Instruments Corporation. (2011, Sep.). Legacy NI ELVIS Replacements and Accessories. [Online]. Available: http://sine.ni.com/nips/cds/view/p/lang/en/nid/207242

[13] Microstick for dsPIC33F and PIC24H Devices, Microchip Technology Inc., Chandler, AZ, 2011. 
[14] Microchip PIC24HJ32GP302/304, PIC24HJ64GPX02/X04 AND PIC24HJ128GPX02/X04 Data Sheet, Microchip Technology Inc., Chandler, AZ, 2007-2011.

[15] .100" [2.54 mm] Contact Centers, Male Breakaway Header Dip Solder/Right Angle/SMT, Sullins Connector Solutions, Inc., San Marcos, CA, 2011.

[16] Pololu Corporation. (2011, Sep.). 270-Point Breadboard. [Online]. Available: http://www.pololu.com/catalog/product $/ 350$

[17] Technical Information - KEYPADSM, Futurlec, New York, NY, 2011.

[18] Microchip MCP23017/MCP23S17 16-Bit I/O Expander with Serial Interface, Microchip Technology Inc., Chandler, AZ, 2011.

[19] Delta Specification for Approval - DC Fan - FFB0412VHN-TP03, Delta Electronics Inc., 2008.

[20] SN754410 Quadruple Half-H Driver Data Sheet, Texas Instruments, Dallas, Texas, 1995.

[21] National Semiconductor LM2940/LM2940C 1A Low Dropout Regulator Data Sheet, National Semiconductor Corp., Santa Clara, CA, 2007.

[22] Maxim +2.5V to +5.5V, Low-Power, Single/Dual, 8-Bit Voltage-Output DACs in $\mu M A X$ Package Data Sheet, Maxim Integrated Products, Sunnyvale, CA, 1997.

[23] Microchip 24AA515/24LC515/24FC515 512K I ${ }^{2} C^{T M}$ CMOS Serial EEPROM Data Sheet, Microchip Technology Inc., Chandler, AZ, 2008.

[24] Character LCD Module Specifications Revision 1.0, Crystalfontz America, Inc., Spokane Valley, WA, 2008.

[25] Advanced Circuits. (2011, Sep.). Advanced Circuits PCB Artist Layout Software. [Online]. Available: http://www.4pcb.com/free-pcb-layout-software/ 